\title{
Measuring the Change in Health-Related Quality of Life in Patients Using Marijuana for Pain Relief
}

\author{
Andrew M. Peterson ${ }^{a} \quad$ Christine Le $^{a} \quad$ Tyler Dautrich $^{b}$ \\ aDepartment of Pharmacy Practice/Pharmacy Administration, University of the Sciences in Philadelphia, Philadelphia, \\ PA, USA; ${ }^{b}$ MoreBetter, Ltd. (dba Releaf App), Hyattsville, MD, USA
}

\section{Keywords}

Health-related quality of life · Pain · Medical cannabis ·

Marijuana

\begin{abstract}
Introduction: Current evidence suggests that cannabinoids are safe with minimal side effects and are effective in managing chronic pain. Data also show that medical marijuana (MM) may improve quality of life (QoL) among patients. However, there are little data showing the health-related QoL (HRQoL) benefit in MM patients using it for pain. The purpose of this study was to determine if there is a relationship between HRQol and MM use in patients using it to relieve pain. Methods: All pain patients aged 18 years or older enrolled in the Pennsylvania MM program were eligible for inclusion. Recruited subjects completed 4 surveys - at enrollment (baseline) then 2, 4, and 8 weeks post-enrollment. We used the EQ-5D survey tool for measuring HRQoL. The primary outcome measure was the change in the EQ-5D Index Score from survey 2 to survey 4 ( 6 week difference). Secondary outcomes included self-reported pain and health scores. Data were analyzed using a paired $t$ test and repeated-measures multivariable analysis to control for both gender and
\end{abstract}

length of time between surveys. Results: 1,762 people responded to the screening request, and 1,393 (79\%) met screening criteria. Of those, 353 (25.3\%) agreed to participate and $51 \%$ completed all 4 surveys, for a final sample of 181 with 85 male and 95 female and one nonbinary subject. The average age was 41.21 (SD = 12.9) years, with no difference between genders. The adjusted HRQoL score improved from 0.722 to 0.747 ( $p=0.011$ ) from survey 2 to survey 4 , as did the self-reported pain and health scores. The EQ-5D subscales revealed no change in mobility or usual activities, significant improvement in anxiety and pain, and a significant worsening in self-care. Conclusion: The results show a significant improvement in HRQoL among patients using MM for pain. The EQ-5D subscales validated the pain improvement and also showed an improvement in anxiety. However, the decline in the self-care subscale may have tempered the overall improvement in $\mathrm{HRQ} \mathrm{L}$, and further research into which aspects of self-care are impacted by MM use in this population is warranted. Overall, there is a positive relationship between MM use and HRQoL in patients using it for pain.

(c) 2021 The Author(s).

Published by S. Karger AG, Basel karger@karger.com www.karger.com/mca

Karger

bOPEN ACCESS
(C) 2021 The Author(s)

Published by S. Karger AG, Basel

This is an Open Access article licensed under the Creative Commons Attribution-NonCommercial-4.0 International License (CC BY-NC) (http://www.karger.com/Services/OpenAccessLicense), applicable to the online version of the article only. Usage and distribution for commercial purposes requires written permission.
Correspondence to:

Andrew M. Peterson, a.peters@ usciences.edu 


\section{Introduction}

The compounds found in the cannabis plant have been used to treat patients with a variety of symptoms and diseases. Current evidence suggests that cannabis is effective in managing chronic pain in adults [1].

While there was a general relaxation in marijuana laws states, the US Drug Enforcement Agency (DEA) still considers marijuana a Schedule I drug under the Controlled Substance Act. As of November 2020, 33 states allow marijuana for medical use and 11 states, plus the district of Columbia, allow for recreational/adult use. In most states, medical marijuana (MM) users are required to register with the state verifying they have a condition in which the state deems appropriate for using marijuana, and then the patient purchases the product through a state-regulated dispensary. States vary in the breadth of conditions that are eligible for treatment with MM. In Pennsylvania, the MM program has 23 approved medical conditions, including pain [2].

There are various types of pain - chronic versus acute and nociceptive versus neuropathic, and identifying and differentiating pain based on the various factors comprising pain is important to aid in treatment choices. However, we do know there is a complex relationship between pain and quality of life (QoL), and it is widely accepted that increased pain leads to decreased QoL and improving pain improves QoL [3,4]. While there is some consensus on the value of marijuana to treat pain [1], there are little data on the impact of such treatment on a patient's QoL. The existing data do show that MM improves QoL among patients with AIDS [5], there are little data on improving QoL in MM patients using it for pain. Further, the adverse effect profile of MM includes impaired executive function, poor job performance, and impaired driving ability among others [6]. These adverse effects may have an impact on QoL and even their health-related QoL (HRQoL). The Center for Disease Control and Prevention (CDC) defines HRQol as "an individual's or group's perceived physical and mental health over time [7]."

In separate cross-sectional studies, both Fiz et al.[8] and Schlienz et al. [9] studied the effect of MM on patients with severe pain. Each study surveyed pain patients, with fibromyalgia as the primary diagnosis, using $\mathrm{MM}$ as part of their treatment. Using tools such as the SF-36 and the World Health Organization Quality of-Life assessment, both found an improvement in the QoL of patients. However, the cross-sectional nature of these studies and the fibromyalgia focus limit the value of these findings and its applicability to other patients.

Health-Related Quality of Life in

Marijuana Pain Patients
Similarly, Boehnke et al. [10] conducted a survey of 244 Michigan cannabis cardholders using cannabis for chronic pain. The survey consisted of 46 questions with a question related to subjects' change in QoL (range $-100 \%$ to $+100 \%)$. Of the 244 participants, 185 completed the survey; 118 were male, 65 were female, and 2 did not answer. The authors reported that patients had a $45 \%$ improvement in QoL; however, the use of an unvalidated HRQoL tool limits the utility of this finding. Further, the authors note that the recall bias inherent in this retrospective survey study is a limitation.

There are few studies examining the impact of $\mathrm{MM}$ on HRQoL [11-14]. However, the studies were cross-sectional, were with patients outside the USA, or did not use validated HRQoL scales, thus leaving a gap in our knowledge about the impact of MM on HRQoL. The purpose of this study was to determine if there is a relationship between HRQol - as estimated from the EQ-5D survey tool - and MM use in Pennsylvania patients using it to relieve their pain.

\section{Materials and Methods}

During the summer and fall of 2020, we conducted a series of surveys of MM users across the state of Pennsylvania using the Qualtrics Survey tool. All material was reviewed and approved by the Institutional Review Board of the University of the Sciences.

\section{Study Population}

All patients enrolled in the Pennsylvania Medical Marijuana program using MM for pain related to one of the qualifying conditions were eligible for inclusion. We did not discriminate patients based on pain type. Those patients $<18$ years of age, not located in Pennsylvania, or were not using MM for one of the qualifying conditions were excluded. We partnered with Releaf App [15] and Keystone Canna Remedies (KCR) to obtain mailing lists of patients using their services. We used this email list to contact participants. KCR has a network of 3 dispensaries, located in Allentown, Bethlehem, and Stroudsburg Pennsylvania.

A convenience sample of patients living in Pennsylvania and using MM identified by Releaf App or patronizing KCR were identified and contacted via email self-reported to either organization. Recruitment flyers were also placed in KCR dispensaries. This flyer contained the study email address in which participants could communicate with the primary investigator directly. Over 12,000 initial emails were sent by Releaf and KCR. The email contained information on the study and a link to a screening survey maintained by the primary investigator. All subsequent communications to participants regarding the study were conducted by the primary investigator with no further contact via the Releaf App. The participant completed the screening survey and their responses determined their continued eligibility. If the subjects met the inclusion/exclusion criteria, they were sent a link to the study website which contained the informed consent. Participants who agreed to the study via the informed consent were then presented with the first of 4 online Pain/HRQoL surveys.

Med Cannabis Cannabinoids 2021;4:114-120 
Table 1. Age and gender distribution of all survey completers

\begin{tabular}{lllll}
\hline & $\begin{array}{l}\text { Males } \\
(n=85)\end{array}$ & $\begin{array}{l}\text { Females } \\
(n=95)\end{array}$ & $\begin{array}{l}\text { Nonbinary } \\
(n=1)\end{array}$ & Total \\
\hline Average age (SD) & $42.48(13.5)$ & $40.28(12.4)$ & 21 & $41.21(12.9)$ \\
\hline
\end{tabular}

Table 2. Difference in outcomes between T1 (survey \#2) and T2 (survey \#4) for all survey completers

\begin{tabular}{|c|c|c|c|c|c|c|c|c|}
\hline & \multicolumn{4}{|l|}{$\mathrm{Raw}^{\mathrm{a}}$} & \multicolumn{4}{|c|}{ Adjusted $^{\mathrm{b}}$} \\
\hline & $\mathrm{T} 1$ & $\mathrm{~T} 2$ & diff & $p$ value & $\mathrm{T} 1$ & $\mathrm{~T} 2$ & diff & $p$ value \\
\hline Pain & 4.204 & 3.735 & -0.469 & 0.008 & 4.194 & 3.711 & -0.483 & 0.008 \\
\hline QoL & 0.721 & 0.746 & 0.025 & 0.011 & 0.722 & 0.747 & 0.025 & 0.011 \\
\hline Health & 69.856 & 73.133 & 3.276 & 0.004 & 69.944 & 73.233 & 3.289 & 0.005 \\
\hline
\end{tabular}

QoL, quality of life; GLM, general linear modeling. ${ }^{\text {a }}$ Paired $t$ test. ${ }^{\mathrm{b}}$ Repeated-measures GLM; days between survey \#2 (T1) and survey \#4 (T2) as covariate.

Respondents were offered a 10\% discount coupon to KCR dispensary for each survey completed and a $20 \%$ discount coupon for completing all 4 surveys. Time limits for use of the coupons were placed to coincide with the administration of the next survey. That is, after completing survey \# 1 , the coupon would expire in 2 weeks, just when the next survey link would be emailed to the participant. Only one coupon per survey per person was allowed. Duplicate survey/repeat surveys were deleted by identifying duplicate emails. KCR only collected the redeemed coupons; they did not maintain any records of the participants.

\section{Survey Tool}

We used an 11-item survey tool consisting of basic demographic questions, a pain scale, and the EQ-5D survey [16]. The National Institute for Health and Care Excellence (NICE) states that the EQ-5D is the preferred measure of health related QoL in adults [17]. The EQ-5D comprises 5 dimensions as follows: mobility, selfcare, usual activities, pain/discomfort, and anxiety/depression and from this creates a HRQoL Index Score. The tool also includes a visual analog scale (VAS) in which respondents self-report their assessment of their health. Besides the NICE recommendation, the EQ-5D was chosen because of its validity and brevity. Five surveys were administered, an initial screening survey and 4 study surveys. The 4 study surveys were administered at baseline, then 2,4 , and 8 weeks later. Baseline refers to the start of the study and not the start of a participant's use of MM. The same 11 questions were administered during all 4 surveys.

Our 3 outcomes were the EQ-5D Index Score, self-reported Pain Score, and self-reported Health Score as measured by the VAS portion of the EQ-5D. The EQ-5D Index Score was a 0-1 scale, the pain score was set to a scale of $0-10$, and the VAS was a $0-100$ scale. The primary outcome measure was change in the EQ-5D Index Score from survey 2 to survey 4 ( 6 week difference). The baseline survey served as a run-in survey to assure the participants would be able to complete the survey and were continuing to use marijuana for pain.
To determine the change in HRQoL in patients using MM for the treatment of pain, we employed a paired $t$ test of respondents who completed all surveys, comparing the EQ-5D Index Score from survey 2 to survey 4 . We also employed the same test to the secondary outcomes, change in the Pain Score and change in the Health Score. To test the relationship between pain relief and HRQoL in patients using MM, we used a repeated-measures general linear modeling analysis, controlling for length of time between surveys and gender. This allowed us to determine if gender differences existed and if the length of time between surveys influenced the outcome. All alpha-values were set at 0.05 .

\section{Sample Size}

Given an estimated standard deviation of 0.5 and an estimated mean EQ-5D utility score of 0.7 , the sample size required to detect the 0.18 difference between baseline and the end of 6 weeks is 62 patients $[18,19]$. To achieve this final number, assuming a $33 \%$ initial response rate and an $80 \%$ retention for each survey, 370 patients needed to meet the screening criteria for an estimated 62 patients at the end of the study.

\section{Results}

The initial email was sent on July 28, 2020, with 1,762 persons responding to the screening request. Of those, 1,393 (79\%) met the screening criteria. Of those, 353 (25.3\%) agreed to participate in the study, as evidenced by reading the consent form and completing the baseline survey. Of the 353 baseline participants, 51\% completed all 4 surveys, for a final sample size of 181 . Table 1 shows the age and genders of the final study population. 
Table 3. Distribution of EQ-5D dimension responses at T1 (survey\#2) and T2 (survey\#4) for all survey completers

\begin{tabular}{|c|c|c|c|}
\hline & Count (\%) T1 & Count (\%) T2 & $p$ value \\
\hline \multicolumn{4}{|l|}{ Mobility } \\
\hline No problems & $110(61)$ & $104(57)$ & \multirow{5}{*}{0.883} \\
\hline Slight problems & $44(24)$ & $56(31)$ & \\
\hline Moderate problems & $19(10)$ & $16(9)$ & \\
\hline Severe problems & $7(4)$ & $5(3)$ & \\
\hline Unable to walk & $1(1)$ & $0(0)$ & \\
\hline \multicolumn{4}{|l|}{ Self-care } \\
\hline No problems & $154(85)$ & $141(78)$ & \multirow{5}{*}{0.006} \\
\hline Slight problems & $20(11)$ & $29(16)$ & \\
\hline Moderate problems & $6(3)$ & $10(6)$ & \\
\hline Severe problems & $1(1)$ & $1(1)$ & \\
\hline Unable to wash or dress & $0(0)$ & $0(0)$ & \\
\hline \multicolumn{4}{|l|}{ Usual activities } \\
\hline No problems & $68(38)$ & $73(40)$ & \multirow{5}{*}{0.854} \\
\hline Slight problems & $74(41)$ & $69(38)$ & \\
\hline Moderate problems & $29(16)$ & $28(15)$ & \\
\hline Severe problems & $8(4)$ & $9(5)$ & \\
\hline Unable to do usual activities & $2(1)$ & $2(1)$ & \\
\hline \multicolumn{4}{|l|}{ Pain/discomfort } \\
\hline No pain/discomfort & $20(11)$ & $32(18)$ & \multirow{5}{*}{$<0.001$} \\
\hline Slight pain/discomfort & $80(44)$ & $81(45)$ & \\
\hline Moderate pain/discomfort & $51(28)$ & $50(28)$ & \\
\hline Severe pain/discomfort & $24(13)$ & $16(9)$ & \\
\hline Extreme pain/discomfort & $6(3)$ & $2(1)$ & \\
\hline \multicolumn{4}{|l|}{ Anxiety/depression } \\
\hline Not anxious/depressed & $49(27)$ & $55(30)$ & \multirow{5}{*}{0.009} \\
\hline Slightly anxious/depressed & $59(33)$ & $68(38)$ & \\
\hline Moderately anxious/depressed & $37(20)$ & $34(19)$ & \\
\hline Severely anxious/depressed & $26(14)$ & $13(7)$ & \\
\hline Extremely anxious/depressed & $10(6)$ & $11(6)$ & \\
\hline
\end{tabular}

Table 2 shows the difference in primary outcome scores between survey \#2 (T1) and survey \#4 (T2). Using the paired $t$ test, the average difference in EQ-5D Index was an improvement of 0.025 and was statistically significantly different $(p=0.011)$. Further, the pain score and health score both improved significantly during the same period. When gender was introduced as a betweensubject variable, there was not a statistically significant relationship to any of the 3 outcome measures.

Table 3 shows the distribution of EQ-5D dimension responses at $\mathrm{T} 1$ and $\mathrm{T} 2$. These results indicate that there was no change in self-reported mobility or usual activities (both $p>0.05$ ), but a significant increase in the number of respondents indicating a worsening of their ability to perform self-care $(p=0.006)$. The results also showed a significant number of respondents indicating an improvement in both pain/discomfort $(p<0.001$ and anxiety/depression $(p=0.009)$.

Health-Related Quality of Life in Marijuana Pain Patients

\section{Discussion}

The overall results indicate that over a 6-week period, chronic MM users had a significant improvement in both pain scores and HRQoL, as measured by the EQ-5D. The EQ-5D QoL dimensions that changed significantly over the 6-week time frame included an improvement in both pain and anxiety scales but a decline in the self-care scale.

Our study showed a small (0.025 U) but statistically significant $(p=0.011)$ improvement in HRQoL using the EQ-5D index. While this value is smaller than the anticipated 0.18 difference, Payakachat suggests that if researchers find changes on the EQ-5D after treatments and significant clinical changes on condition-specific measures, the EQ-5D changes can be considered responsive [20]. It is difficult to interpret what a 0.025 change in the EQ-5D Health Index is but that a positive change occurred, along with a positive change in the pain score and 
VAS score, underlie the notion that there was an improvement in this group of patient's HRQoL.

Upon further examination of the components that comprise the EQ-5D Health Index, one sees that the improvements to the pain and anxiety dimensions are tempered by a decline in the area of self-care. This is important because the side effect profile of cannabis may be diminishing the improvement in HRQoL. While we did not examine the doses used nor the composition of the products, all products sold in the MM program of Pennsylvania are marijuana, thus containing some level of THC. We suggest further investigation of the dose-response relationship between THC and HRQoL to see if there is an optimal balance between pain/anxiety improvement and self-care based on dose.

A review of the literature found no other study connecting the use of marijuana with declines in self-care in pain patients using MM. There are many sources describing the negative consequences of marijuana, using a variety of scales [21], and the Marijuana Consequences Questionnaire (MACQ) [22] does contain a 9-question selfcare domain evaluating self-care. In this domain are questions related to eating, activity, physical appearance, mental acuity, and energy/motivation. Given that our study found a decline in self-care among pain patients using $M M$, it would be of interest to learn what aspects of self-care change when using MM for pain.

Haroutounian et al. [11] conducted a longitudinal study in 176 patients over 6 months using the Treatment Outcomes in Pain Survey - Short Form (S-TOPS) questionnaire. Their results showed that patients' pain severity score (1-10 scale) decreased from a 7.5 to 6.25 and a statistically significant improvement in select QoL measures embedded in the S-TOPS. These measures included the Family-Social Disability and Role-Emotional Disability scores, but they did not find a significant change in the physical disability scores over time. The measures used in our study are not directly comparable to those used in the Haroutounian study, but their finding of no change in physical disability scores is consistent with our finding of no change in mobility scores using the EQ-5D.

Safakish and colleagues [23] studied plant-based cannabis treatment in patients with severe pain. In a 12-month observational study, the authors found that cannabis treatment was associated with improvements in pain severity at month 1 and sustained over the 12-month observation period $(p<0.001)$. Further, they noted significant improvements in physical and mental health at 3 months, as measured by the SF-12 $(p<0.002)$. The 1 -month and then 12 -month pain relief findings of this study are consistent with our pain relief findings at 6 weeks. Further, our pain relief improvement is corroborated by 2 pain relief measures - both by a $0-10$ scale and the EQ-5D pain/discomfort scale, as both were significantly improved after 6 weeks of persistent use of marijuana. Further, our findings are consistent with Safakish's QoL findings - though our findings show an improvement at 6 weeks versus 3 months.

\section{Limitations}

One of the primary limitations to this study is the convenience sampling method coupled with the incentive program employed. The sample was drawn from 2 sources of patients; the Releaf App - where pain patients all across Pennsylvania were contacted and KCR - a trio of dispensaries located in northeast Pennsylvania. While we did not attempt to characterize the geographic distribution of the sample, the respondents were most likely from the northeast Pennsylvania area since the incentive to participate was geographically centered in that area. This may limit the generalizability of the results to other patients throughout the state or elsewhere. Future studies should use a more geographically diverse population and a different incentive program to assure a greater representation of the population. Further, we included all users of MM, recognizing that those naive users may have a different outcome than experienced users, which also limits the generalizability of the data. Future studies will attempt to stratify those new versus persistent users.

Another related limitation is the loss to follow-up. While nearly 1,400 people were eligible, just over 350 people enrolled in the study and only about one-half of those completed all 4 surveys. Those persons dropping out may have had poor pain management and/or a decline in their HRQoL between surveys that this study would not have captured. We did not collect sufficient demographic data to compare the completers versus the noncompleters; thus, we could not determine how representative this sample is to the initial population. A future study including more specific information such race/ethnicity, socioeconomic status, location, marital status, and education would help characterize the population better.

And last, while we did not examine the doses used nor the composition of the products, all products sold in the MM program of Pennsylvania are marijuana, thus containing some level of THC. A future study collecting specific product information such as dosing, route of administration, and THC and other cannabinoid concentrations would help in determining the impact of such factors on HRQoL.

This is one of the first studies to examine a longitudinal view of HRQoL in patients using MM for pain using the 
EQ-5D tool. The results of this study show that MM, when used for the treatment of pain, can be beneficial at improving a patient's QoL along with alleviating their pain. However, we noted the improvement in HRQoL is primarily due to an improvement in pain and anxiety, but the self-care dimension declined. Further examination of the impact of MM on self-care is necessary.

\section{Acknowledgements}

The authors of this study would like to acknowledge the support and assistance of Victor Guadagnino, founder of Keystone Canna Remedies and his team. They were integral in recruiting and supporting the subjects involved in this study.

\section{Statement of Ethics}

All the materials were reviewed and approved by the Institutional Review Board of the University of the Sciences (Protocol\# 1619322-1), and all subjects eligible for participation were consented in writing, electronically, and given the opportunity to withdraw from the study at any time without consequence.

\section{Conflict of Interest Statement}

Dr. Andrew Peterson is on the Medical Advisory Board for Holistic Industries, a cannabis dispensary company seeking licensure in the state of New Jersey. He also serves as the executive director of the Substance Use Disorders Institute at University of the Sciences, which offers a 4-h Pennsylvania Department of Health-approved MM education program. Christine Le has no conflicts to declare. Tyler Dautrich has no conflicts to declare.

\section{Funding Sources}

Keystone Canna Remedies (KCR) Dispensary provided discount coupons to subjects participating in this study. KCR did not have a role in the design of this study or the interpretation of the data and results. No other funding or sponsorship was used.

\section{Author Contributions}

Andrew M. Peterson contributed to conception of and design of study; analysis and interpretation of the work; drafting the work; and final approval. Christine Le contributed to analysis and interpretation of the data and final approval. Tyler Dautrich contributed to conception of and design of study and final approval.

\section{Data Availability Statement}

All data generated or analyzed during this study are included in this article. The de-identified dataset may be obtained and/or further enquires may be directed to the corresponding author, A.M.P.

\section{References}

1 National Academies of Sciences, Engineering, and Medicine. The health effects of cannabis and cannabinoids: the current state of evidence and recommendations for research. Washington (DC): The National Academies Press; 2017

2 Pennsylvania Medical Marijuana Program. Available from: https://www.health.pa.gov/ topics/programs/Medical\%20Marijuana/ Pages/Medical\%20Marijuana.aspx Viewed 2019 Nov 19

3 Hadi MA, McHugh GA, Closs SJ. Impact of chronic pain on patients' quality of life: a comparative mixed-methods study. J Patient Exp. 2019 Jun;6(2):133-41. Epub 2018 Jul 5. Pubmed PMID: 31218259; PubMed Central PMCID: PMC6558939.

4 Katz N. The impact of pain management on quality of life. J Pain Symptom Manage. 2002 Jul;24(1 Suppl 1):S38-47. Pubmed PMID: 12204486 .
5 D'Souza G, Matson PA, Grady CD, Nahvi S, Merenstein D, Weber KM, et al. Medicinal and recreational marijuana use among HIVinfected women in the women's interagency HIV study (WIHS) cohort, 1994-2010. J Acquir Immune Defic Syndr. 2012;61:618-26.

6 Feeney KE, Kampman KM. Adverse effects of marijuana use. Linacre Q. 2016;83(2):174-8.

7 Centers for Disease Control and Prevention. Measuring healthy days: population assessment of health-related quality of life. Atlanta (Georgia): Centers for Disease Control and Prevention; 2000.

8 Fiz J, Durán M, Capellà D, Carbonell J, Farré M. Cannabis use in patients with fibromyalgia: effect on symptoms relief and health-related quality of life. PLoS One. 2011;6(4): e18440.

9 Schlienz NJ, Scalsky R, Martin EL, Jackson H, Munson J, Strickland JC, et al. A cross-sectional and prospective comparison of medicinal cannabis users and controls on self-reported health. Cannabis Cannabinoid Res. 2020. Epub 2020 Jun 8.
10 Boehnke KF, Litinas E, Clauw DJ. Medical cannabis use is associated with decreased opiate medication use in a retrospective crosssectional survey of patients with chronic pain. J Pain. 2016 Jun;17(6):739-44. Epub 2016 Mar 19. Pubmed PMID: 27001005.

11 Haroutiunian S, Ratz Y, Ginosar Y, Furmanov K, Saifi F, Meidan R, et al. The effect of medicinal cannabis on pain and quality-oflife outcomes in chronic pain: a prospective open-label study. Clin J Pain. 2016 Dec; 32(12):1036-43.

12 Ware MA, Wang T, Shapiro S, Robinson A, Ducruet T, Huynh T, et al. Smoked cannabis for chronic neuropathic pain: a randomized controlled trial. CMAJ. 2010 Oct 5;182(14): E694-701. Epub 2010 Aug 30.

13 Goldenberg M, IsHak WW, Danovitch I. Quality of life and recreational cannabis use. Am J Addict. 2017;26(1):8-25.

14 Gulbransen G, Xu W, Arroll B. Cannabidiol prescription in clinical practice: an audit on the first 400 patients in New Zealand. BJGP Open. 2020 May 1;4(1):bjgpopen20X101010. 
15 RELEAF. Available from: https://releafapp. com/about-us/ (viewed 2019 Nov 19).

16 EQ-5D Health-related Quality of Life Tool. Available from: https://euroqol.org/eq-5dinstruments/eq-5d-5l-about/.

17 NICE. Guide to the methods of technology appraisal 2013. National Institute for Health and Care Excellence; 2013. Available from: https: //www.ncbi.nlm.nih.gov/books/ NBK395867/pdf/Bookshelf_NBK395867.pdf [2021 Jan].

18 Torrance N, Lawson KD, Afolabi E, Bennett MI, Serpell MG, Dunn KM, et al. Estimating the burden of disease in chronic pain with and without neuropathic characteristics: does the choice between the EQ-5D and SF-6D matter? Pain. 2014;155(10):1996-2004.

19 Coretti S, Ruggeri M, McNamee P. The minimum clinically important difference for EQ5D index: a critical review. Expert Rev Pharmacoecon Outcomes Res. 2014 Apr;14(2): 221-33.

20 Payakachat N, Ali MM, Tilford JM. Can the EQ-5D detect meaningful change? A systematic review. PharmacoEconomics. 2015; 33(11):1137-54.

21 Pearson MR. A meta-analytic investigation of the associations between cannabis use and cannabis-related negative consequences. Psychol Addict Behav. 2019;33(3):190-6.

22 Simons JS, Dvorak RD, Merrill JE, Read JP. Dimensions and severity of marijuana consequences: development and validation of the marijuana consequences questionnaire (MACQ). Addict Behav. 2012 May;37(5): 613-21.

23 Safakish R, Ko G, Salimpour V, Hendin B, Sohanpal I, Loheswaran G, et al. Medical cannabis for the management of pain and quality of life in chronic pain patients: a prospective observational study. Pain Med. 2020 Jun 18; 21(11):3073-86. 